\title{
Screening drug target combinations in disease-related molecular networks
}

\author{
Min Luo, Jianfeng Jiao and Ruiqi Wang ${ }^{*}$ \\ From The 12th International Conference on Computational Systems Biology (ISB 2018) \\ Guiyang, China. 18-21 August 2018
}

\begin{abstract}
Background: For treating a complex disease such as cancer, some effective means are needed to control biological networks that underlies the disease. The one-target one-drug paradigm has been the dominating drug discovery approach in the past decades. Compared to single target-based drugs, combination drug targets may overcome many limitations of single drug target and achieve a more effective and safer control of the disease. Most of existing combination drug targets are developed based on clinical experience or text-and-trial strategy, which cannot provide theoretical guidelines for designing and screening effective drug combinations. Therefore, systematic identification of multiple drug targets and optimal intervention strategy needs to be developed.

Results: We developed a strategy to screen the synergistic combinations of two drug targets in disease networks based on the classification of single drug targets. The method tried to identify the sensitivity of single intervention and then the combination of multiple interventions that can restore the disease network to a desired normal state. In our strategy of screening drug target combinations, we first classified all drug targets into sensitive and insensitive single drug targets. Then, we identified the synergistic and antagonistic of drug target combinations, including the combinations of sensitive drug targets, the combinations of insensitive drug target and the combination of sensitive and insensitive targets. Finally, we applied our strategy to Arachidonic Acid (AA) metabolic network and found 18 pairs of synergistic drug target combinations, five of which have been proven to be viable by biological or medical experiments.

Conclusions: Different from traditional methods for judging drug synergy and antagonism, we propose the framework of how to enhance the efficiency by perturbing two sensitive targets in a combinatorial way, how to decrease the drug dose and therefore its side effect and cost by perturbing combinatorially a main sensitive target and an auxiliary insensitive target, and how to perturb two insensitive targets to realize the transition from a disease state to a healthy one which cannot be realized by perturbing each insensitive target alone. Although the idea is mainly applied to an AA metabolic network, the strategy holds for more general molecular networks such as combinatorial regulation in gene regulatory networks.
\end{abstract}

Keywords: Drug target combination, Disease network, Combination index, Biology system

\section{Background}

In the past decades, effective treatments of a complex diseases require practical means to control the biological networks underlying the disease. Biological networks are often robust to external disturbances, so it is difficult to control network dynamics by controlling

*Correspondence: rqwang@shu.edu.cn

Department of Mathematics, Shanghai University, No.99, Shangda Road, Shanghai, China a single target, and drugs targeting multiple targets may counter these troubles [1]. It has been long realized that the behavior of drug molecules in a disease network can be complex. The one-target onedrug paradigm has been the dominating drug discovery approach which result in many drugs marketed but cannot treat certain complex diseases sufficiently [2-4]. In fact, drug combination therapeutics are often more effective and are used to treat various complex diseases

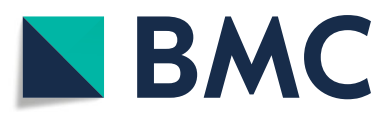

(c) The Author(s). 2019 Open Access This article is distributed under the terms of the Creative Commons Attribution 4.0 International License (http://creativecommons.org/licenses/by/4.0/), which permits unrestricted use, distribution, and reproduction in any medium, provided you give appropriate credit to the original author(s) and the source, provide a link to the Creative Commons license, and indicate if changes were made. The Creative Commons Public Domain Dedication waiver (http://creativecommons.org/publicdomain/zero/1.0/) applies to the data made available in this article, unless otherwise stated. 
in recent years. To overcome the limitations of the singletarget-based drugs, growing attention has been paid to drug discoveries involving multiple targets at the level of disease networks [5]. Actually, it has been a long history of using combination drugs to treat diseases. For example, the Traditional Chinese Medicines (TCM), especially herbal medicines, which can be viewed as the combinations of multiple compounds with synergy effects, have been used for thousands of years [6].

A diverse range of works have been carried out in the emerging field of multi-target drug design, many methods have been proposed to identify effective drug combinations [5, 7-11]. It is recommended that system-oriented drug design should consider the intrinsic properties of biological systems, such as robustness [12]. Many mathematical models of disease-relevant pathways have been constructed which have the potential to elucidate underlying mechanisms of diseases and to identify treatment strategies $[13,14]$. And analyzing the properties of networks can attribute to identify potential drug targets and understand the connectivity between them $[15,16]$.

There may be many beneficial outcomes of synergy, such as increasing the therapeutic effect, reducing the dose but increasing or maintaining the same efficacy to avoid toxicity or reducing the cost of the drug, minimizing or slowing the development of drug resistance. For these therapeutic benefits, drug combinations have been widely used and become the leading choice for treating the most dreadful diseases, such as cancers and infectious diseases.

In this paper, we developed a strategy to screen multiply drug targets combinations. For a given disease network, we tried to identify effective points and the combination of interventions that can restore the disease network to a desired normal state. In our strategy, we first classified all drug targets into sensitive and insensitive single drug targets. Then, we identified synergy and antagonism for all drug target combinations which include the combinations of sensitive drug targets, the combinations of insensitive drug targets, and the combinations of sensitive and insensitive drug targets. We applied our strategy to the arachidonic acid (AA) metabolic network and we found 18 pairs of synergistic drug target combinations, five of which have been proven to be viable through biological or medical experiments.

\section{Results}

\section{The statement of screening drug target combinations}

Feedback loops, cross-talk and other network-intrinsic properties can make the effects of drug molecules much more complicated than predicted by a linear one-drug one-target approach [17]. We here develop a strategy to screen synergetic drug target combinations with pre-determined therapeutic effects. For convenience, two network states are defined in the disease network: the disease state and the normal state (or desired state). The disease state is a network state which the production of disease-related molecules is abnormal. The normal state is the network state which one would like to achieve after taking medicine. The main procedure of screening the synergetic drug target combinations is to perturb the network and optimize it toward the normal state.

\section{Determining the sensitive and insensitive individual drug targets}

Before screening the synergetic drug target combinations, we need to identify the sensitive and insensitive individual drug targets. All single drug targets are ranked according to a criterion that measures their potential in restoring the network state when they are perturbed. In other words, we can rank the individual drug targets according to their sensitivities. The process of determining the sensitive and insensitive individual drug targets includes the following steps:

Step 1: Define the disease and normal states. A state can be defined as a steady state or transient of a network, which can be a collection of concentrations of proteins or metabolites. Generally, the disease state is a steady state of a disease network under standard parameters. The normal state is the desired state after perturbations.

Step 2: Select reactions that can be controlled by drugs. For example, if the drug targets are enzymes, conveniently, we mark all drug targets with $d_{1}, \cdots, d_{i}, \cdots, d_{n}$, and mark the initial concentration of the $i$-th enzyme with $E_{d i}$.

Step 3: Change the concentration of the selected target enzyme until the network is translated into the normal state. We use $E_{h i}$ to mark the concentration of the $i$-th enzyme after changing.

Step 4: Define the degree of change in concentration of each enzyme as $\triangle E_{i} / E_{d i}$, where $\triangle E_{i}=\left|E_{d i}-E_{h i}\right|$. Rank the value of $\triangle E_{i} / E_{d i}$ from small to large. Then, we can have an order of drug targets, as shown in Table 1.

We define a threshold of $a$ and $a$ is a constant. An enzyme which satisfies the condition $\triangle E_{i} / E_{d i} \leq a$ is defined as a sensitive drug target, and an insensitive drug target otherwise.

Table 1 Ranking the value of $\frac{\Delta E_{i}}{E_{d i}}$ from small to large

\begin{tabular}{lll}
\hline Number & Enzyme & $\Delta E_{i} / E_{d i}$ \\
\hline 1 & $d_{1}$ & $\Delta E_{1} / E_{d 1}<1$ \\
$\vdots$ & $\vdots$ & $\vdots$ \\
$j$ & $d_{j}$ & $\Delta E_{j} / E_{d j} \leq 1$ \\
$\vdots$ & $\vdots$ & $\vdots$ \\
$n$ & $d_{j}$ & $\Delta E_{j} / E_{d j}>1$ \\
\hline
\end{tabular}




\section{Screening synergistic drug target combinations Identifying the synergistic combinations of sensitive drug targets}

With the development of medicine science and pharmacology industry, combinatorial drugs are becoming the standard to cure many complex diseases [18]. As a result, some methods have been proposed to identify effective drug combinations. Combination index $(\mathrm{CI})$ analysis is widely used to evaluate drug interactions in combination drug disease treatment. The Loewe additivity model has been widely used when the combined effect of two drugs is additive. The model can be written as:

$$
\frac{(D)_{1}}{(D x)_{1}}+\frac{(D)_{2}}{(D x)_{2}}=1 \text {, }
$$

where $(D)_{1}$ and $(D)_{2}$ are the respective combinatorial doses of drug 1 and drug 2 , and $(D x)_{1}$ and $(D x)_{2}$ are the corresponding single doses for drug 1 and drug 2 with the same effect. When (1) holds, it can be concluded that the combinatorial effect of the two drugs is additive. Based on (1), the CI can be defined as:

$$
C I=\frac{(D)_{1}}{(D x)_{1}}+\frac{(D)_{2}}{(D x)_{2}} \text {. }
$$

According to (2), the CI can be used to classify drug interactions as synergistic, additive, antagonistic and hybrid [19]. And the corresponding curves are shown in [20].

1. Synergy: $C I<1$. In this case, the effect of the drug combination is superior to each single drug, for example, the combined drugs allow each drug to have a smaller dose. Therefore, the synergistic combination of drugs can exhibit fewer side effects in the treatment of diseases.

2. Antagonism: $C I>1$. In this case, the effect of each single drug is superior to the drug combination, and the combined drugs may require each drug to have more dose. This may lead to increased drug costs and greater side effects.

3. Additivity: $C I=1$. In this case, the efficiency of the combinatorial drugs is equal to the efficiency of the single drug. At the same time, this additivity type also provides a standard for judging synergistic and antagonistic combinations.

4. Hybrid. For a drug combination, some drug dose combinations $\left(\frac{(D)_{1}{ }^{\prime}}{(D x)_{1}}, \frac{(D)_{2}}{(D x)_{2}}\right)$ satisfy $C I>1$, while other drug dose combinations $\left(\frac{(D)_{1}}{(D x)_{1}}, \frac{(D)_{2}}{(D x)_{2}}{ }^{\prime \prime}\right)$ satisfy $C I<1$. That is to say, this drug combination can have synergistic or antagonistic effects, depending the drug doses of the combination.

In the past, Chou and co-workers have proposed semiquantitative methods for describing the degrees of synergism or antagonism [6]. These methods are now expanded as shown in Table 2 [21]. Using the CI grading, synergism is subdivided into nearly additive, slight
Table 2 Description and symbols of synergism or antagonism in drug target combination studies analyzed with the $\mathrm{Cl}$ method

\begin{tabular}{llc}
\hline Range of Combination Index & Description & Graded Symbols \\
\hline$<0.1$ & very strong synergism & +++++ \\
$0.1-0.3$ & strong synergism & ++++ \\
$0.3-0.7$ & synergism & +++ \\
$0.7-0.85$ & moderate synergism & ++ \\
$0.85-0.90$ & slight synergism & + \\
$0.90-1.10$ & nearly additive & +- \\
$1.10-1.20$ & slight antagonism & - \\
$1.20-1.45$ & moderate antagonism & -- \\
$1.45-3.3$ & antagonism & -- \\
$3.3-10$ & strong antagonism & --- \\
$>10$ & very strong antagonism & ---- \\
\hline
\end{tabular}

synergism, moderate synergism, synergism, strong synergism, and very strong synergism and antagonism is divided by a similar way [21]. We mainly concern about the qualitative shape of the isobolograms for correctly identifying the drug pair categories, and use the smallest or largest $\mathrm{CI}$ of all drug dose combinations as the $\mathrm{CI}$ for this drug pair [22].

In recent years, the development of disease types is very rapid, but the development of drugs is far slow than the development of diseases which leads to the case that there are no suitable drugs to cure many complex diseases. It is very difficult to find a new drug, which inspires us to find new combination of old drugs. Based on this biological significance, we try to study the combinations of sensitive drug targets.

For all drug target combinations of sensitive drug targets, we analyze the synergy and antagonism of the sensitive drug target combination by computing CI according to the doses of drugs. What's more, we rank the degrees of synergism or antagonism of all drug target combinations according to Table 2.

Identifying the synergistic combinations of insensitive targets In biomedical, when the two drugs are used alone, they can not cure a disease, but when the two drugs are used in combination, the disease can be cured or the effect can be obviously improved. The combination of insensitive drug targets may provided an idea for solving such problems.

For the insensitive drug targets, we combine them in pairs and calculate $\Delta E_{i} / E_{d i}$ where $\Delta E_{i}=\left|E_{d i}-E_{h i}\right|$. The drug combinations which $\triangle E_{i} / E_{d i} \leq 1$ needs to be satisfied for the perturbation to insensitive targets can guarantee that the doses of drugs are used less than those 
used before combination. So, if each drug target of a combination satisfy $\triangle E_{i} / E_{d i} \leq 1$, then this combination is defined as synergistic combinations.

\section{Identifying the synergistic combinations of sensitive and insensitive drug targets}

In clinical treatment, many diseases do not have a suitable treatment because the used drugs are relatively expensive. If the dosage of expensive drugs can be reduced with the auxiliary of cheap drugs, a better treatment strategy is able to be designed. Based on the above ideas, the combinations of sensitive and insensitive drug targets may provide a feasible method.

For the combination of the sensitive and insensitive drug targets, we calculate the $\Delta E_{i} / E_{d i}$ of sensitive and insensitive drug targets respectively.

For the sensitive drug target, we define a constant $\alpha$ and set the following formula:

$$
\frac{\left(\frac{\Delta E_{i}}{E_{d i}}\right)^{1}}{\left(\frac{\Delta E_{i}}{E_{d i}}\right)^{2}}=\frac{\left(\triangle E_{i}\right)^{1}}{\left(\triangle E_{i}\right)^{2}}=\alpha
$$

where $\left(\triangle E_{i} / E_{d i}\right)^{1}$ is the value of $\triangle E_{i} / E_{d i}$ after combination and $\left(\triangle E_{i} / E_{d i}\right)^{2}$ is the value of $\triangle E_{i} / E_{d i}$ before combination. $\alpha$ can be determined in advance and satisfy $0<\alpha<1$.

Then, we find the drug target combination which insensitive drug targets satisfy $\triangle E_{i} / E_{d i} \leq 1$ and the sensitive drug targets is $\left(\triangle E_{i}\right)^{1} /\left(\triangle E_{i}\right)^{2}=\alpha$. Such combinations are defined as synergistic.

The principle of synergistic combinations of sensitive and insensitive drug targets is reducing the dosage of drugs perturbed to sensitive target by the auxiliary drug, i.e., the perturbation to insensitive drug target. The drug combinations in which $\Delta E_{i} / E_{d i} \leq 1$ needs to be satisfied for the perturbation to insensitive targets and $\left(\triangle E_{j}\right)^{1} /\left(\triangle E_{j}\right)^{2}=\alpha(0<\alpha<1)$ for the sensitive target can guarantee that the doses of drugs are used less than those used before combination. In this case, the drug combinations can improve the efficacy and reduce the doses of drugs and their side effects. For example, for a combination of sensitive and insensitive drug targets, if the perturbation to the sensitive drug target satisfies $\left(\triangle E_{j}\right)^{1} \ll\left(\triangle E_{j}\right)^{2}$, and the cost of drug for the insensitive target is relatively low, then such a perturbation combination may have more advantages.

\section{Applying the strategy of screening drug target combinations to AA metabolic network The metabolic network of AA in human PMNs}

Now, we apply our strategy to the Arachidonic Acid (AA) metabolic network. There have been some stud- ies on drug combinations of AA metabolic network [23]. Inflammation is a type of nonspecific immune response to infection, irritation, or other injury. It is characterized by redness, swelling, pain, and some loss of function [24]. Many key enzymes involved in AA metabolic network are responsible for generating inflammation mediators. The AA metabolic network with a multi-cellular ensemble of human polymorphonuclear leukocytes (PMNs), endothelial (EC) and platelet (PLT) cells. Extensive researches on the metabolism of AA metabolic network in human PMNs have been performed. Leukotrienes (LTs) LTs are the major inflammatory mediators produced in PMN [25]. In this paper, we study the dynamic properties of the AA metabolic network in human PMNs, as shown Fig. 1, to gain more insights into anti-inflammatory drug target design. Ordinary differential equations (ODEs) are constructed to simulate the its dynamics, where 24 equations were constructed (see Additional file 1). All the parameters of the ODEs are shown in Additional file 2.

\section{Determining the sensitive and insensitive individual drug} targets in AA metabolic network

It is showed that LTs rather than prostaglandins (PGs) are the main inflammatory mediators produced in human PMNs [17]. Here, the disease state of the AA network is defined as a state where the output of LTs is markedly above the normal level. Since the standard parameters fitted correspond to the abnormal metabolism of LTs, the stable state under standard parameters is described as the disease state. The desired state after perturbation is defined as low output of inflammatory mediators, that is, the cumulative output of LTB4 should be smaller $10 \%$ than that in the disease state. Since it is difficult to give a definite cutoff to distinguish between normal and disease states and the threshold is set to be $10 \%$. Eight enzymes in the AA metabolic network are selected as drug targets, because perturbing them individually can induce the transition of the AA metabolic network from a disease state to a normal one $[3,17]$.

The process of determining the sensitive and insensitive individual drug targets in AA metabolic network includes the following steps:

Step 1: Define the disease and normal states. The normal state is defined as a state where $1 \mathrm{~h}$ cumulative production of LTB4 is less than 10\% of that in the disease state. The fluxes of other metabolites are not monitored.

Step 2: Eight enzymes are chosen as drug targets (as shown in Table 3). We mark the initial concentration of the $i$-th enzyme with $E_{d i}$.

Step 3: Change the concentration of each target enzyme until the $1 \mathrm{~h}$ cumulative production of LTB4 is just below 


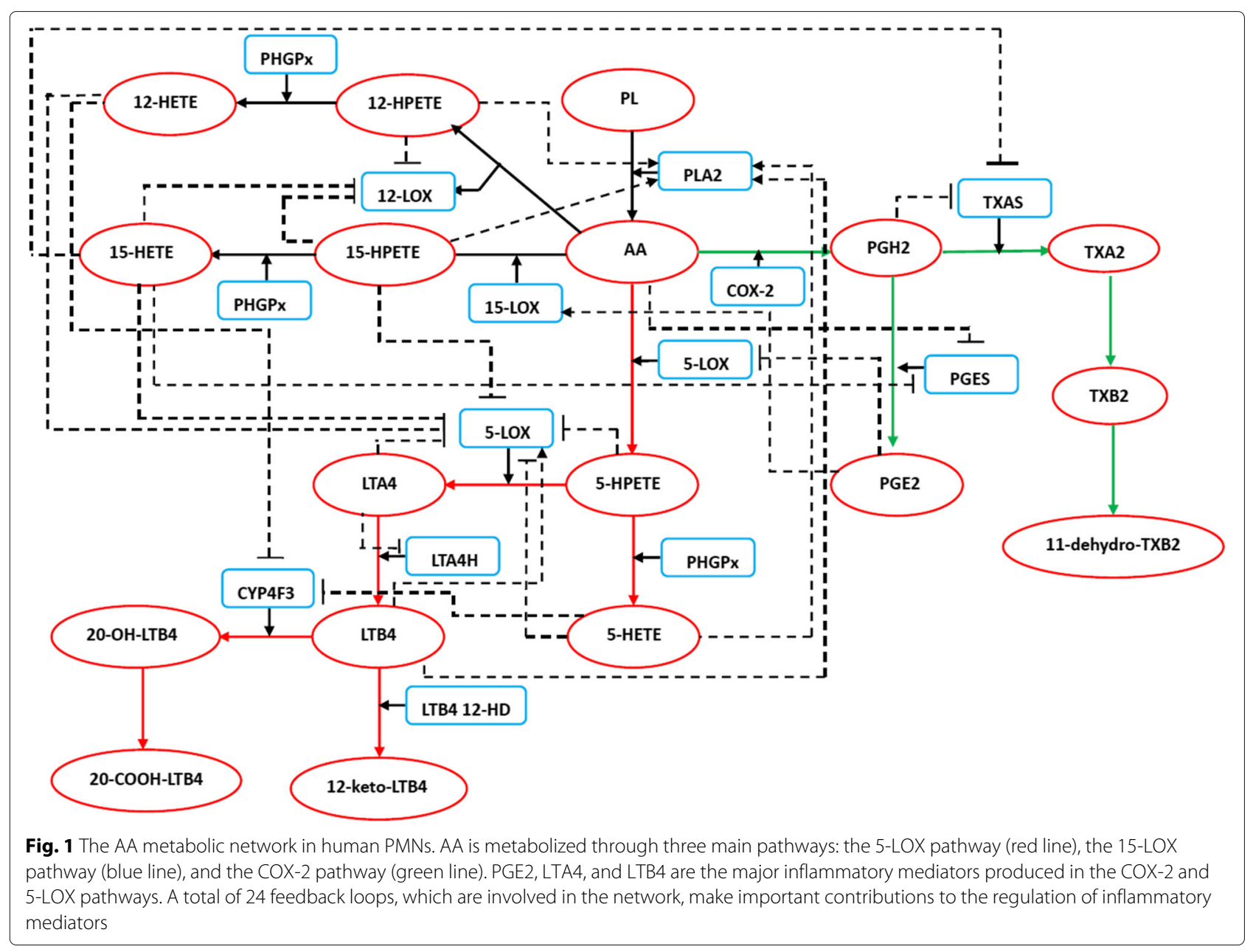

$10 \%$ of that in the disease state. After $1 \mathrm{~h}$, we mark the changed concentration of the $i$-th enzyme with $E_{h i}$.

Step 4: Define the degree of change in concentration of each enzyme as $\Delta E_{i} / E_{d i}$, where $\Delta E_{i}=\left|E_{d i}-E_{h i}\right|$, and rank the value of $\triangle E_{i} / E_{d i}$ according to the degree.

To ensure the sensitivity of drug targets, we set $a=$ 1, i.e, when the transition is realized, the perturbation quantity is equal to or less than the initial concentration of the selected target. Then, four enzymes which satisfy $\triangle E_{i} / E_{d i} \leq 1$ can be selected as sensitive drug targets and

Table 3 Ranking the value of $\frac{\Delta E_{i}}{E_{d i}}$ from small to large

\begin{tabular}{lll}
\hline Number & Enzyme & $\Delta E_{i} / E_{d i}$ \\
\hline 1 & CYP4F3 & 0.0769 \\
2 & PLA2 & 0.1979 \\
3 & PHGPX & 0.213043 \\
4 & TXAS & 0.99999 \\
5 & 15-LOX & 43.6388 \\
6 & 5-LOX & 135.4 \\
7 & LTA4H & 15066606.44 \\
8 & 12-LOX & $1.52 \times 10^{17}$ \\
\hline
\end{tabular}

the remaining four enzymes are insensitive, as shown in Table 3.

\section{Screening synergistic drug target combinations in AA metabolic network \\ Identifying the synergistic combinations of sensitive drug targets}

We use CI to identify the synergistic and antagonistic combinations of sensitive drug targets. According to Eq. 2, we calculate CI value of every combination of sensitive drug targets. We mainly concern about the qualitative shape of the CI isobolograms for correctly identifying synergistic drug pairs, and use the smallest or largest CI of all dose combinations as the $\mathrm{CI}$ for one drug target pair. The $\mathrm{CI}$ isobolograms of each combination of sensitive drug targets are shown in Fig. 2.

We rank the combinations of sensitive drug targets by the value of $\mathrm{CI}$ and the degrees of synergism or antagonism according to Table 2, as shown in Table 4. It is obviously that there are four pairs of combinations that have synergistic effects, and three pairs of combinations have antagonistic effects. Especially, the combination of 

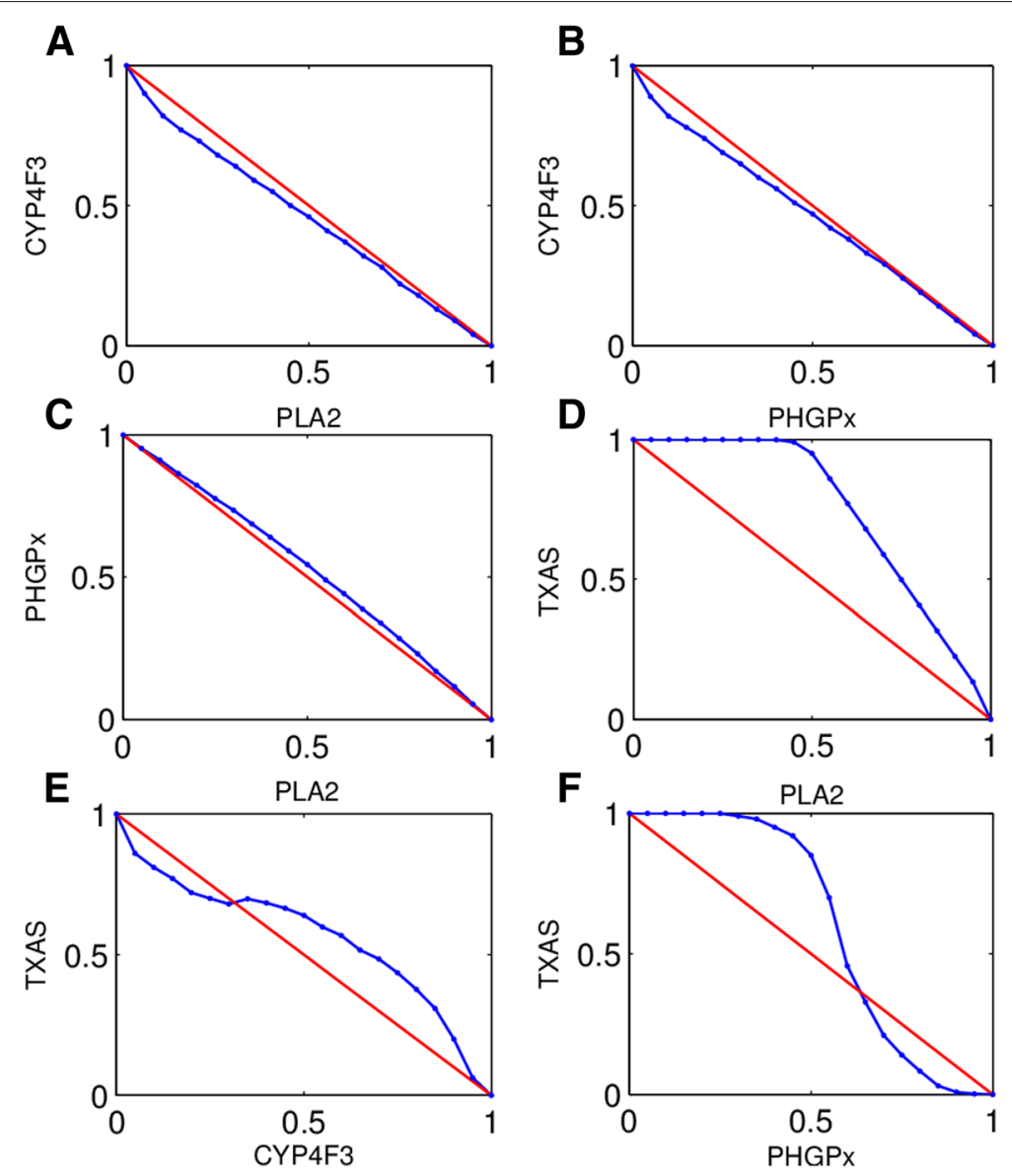

Fig. 2 The result of identifying the synergistic and antagonistic combinations of sensitive drug targets. In this figure, there are three type curves in this figure. The points represent different drug doses. $\mathbf{a}$ and $\mathbf{b}$ are the synergistic combinations. $\mathbf{c}$ and $\mathbf{d}$ are antagonism combinations. $\mathbf{e}$ and $\mathbf{f}$ are hybrid type

TXAS and CYP4F3 is the type of hybrid. For different drug dose combinations, TXAS and CYP4F3 may have synergy or antagonism.

Identifying the synergistic combinations of insensitive targets There are four insensitive drug targets which satisfy $\triangle E_{i} / E_{d i}>1$. Now, we combine them in pairs and calculate the value of $\triangle E_{i} / E_{d i}$ after combination, as shown in Fig. 3. It is obviously that two combinations satisfy the condition $\triangle E_{i} / E_{d i} \leq 1$. The two combinations are 5-LOX, LTA4H and 5-LOX, 12-LOX. According to our strategy, these two combinations are synergistic drug target combinations. In other words, these two pair targets are synergistic combinations and we can perturb them

Table 4 Description and symbol of synergy or antagonism in drug target combination of sensitive drug targets studies analyzed by $\mathrm{Cl}$ method

\begin{tabular}{llll}
\hline Combination name & Value of Combination Index & Description & Graded Symbols \\
\hline PHGPX-TXAS & 0.8806 & Slight synergism & + \\
TXAS-CYP4F3 & 0.91 & Nearly additive & +- \\
PLA2-CYP4F3 & 0.92 & Nearly additive & +- \\
PHGPX-CYP4F3 & 0.92 & Nearly additive & +- \\
PLA2-PHGPX & 1.0442 & Nearly additive & +- \\
TXAS-CYP4F3 & 1.1850 & Slight antagonism & - \\
PLA2-TXAS & 1.45 & Antagonism &.- \\
\hline
\end{tabular}




\begin{tabular}{|c|c|c|c|}
\hline Enzyme 1 & Enzyme 2 & $\frac{\Delta \mathrm{E}_{1}}{\mathrm{E}_{\mathrm{d} 1}}$ & $\frac{\Delta \mathrm{E}_{2}}{\mathrm{E}_{\mathrm{d} 2}}$ \\
\hline 15-LOX & LTA4H & 6.5 & 2 \\
\hline 15-LOX & $12-$-LOX & 0.347 & $1.5 \times 10^{10}$ \\
\hline LTA4H & $12-$ LOX & $1.5 \times 10^{7}$ & $1.5 \times 10^{17}$ \\
\hline 5-LOX & $15-$ LOX & 6.8 & 1 \\
\hline 5-LOX & LTA4H & 0.167 & 0.799 \\
\hline 5-LOX & 12-LOX & 0.93 & 0.666 \\
\hline
\end{tabular}

Fig. 3 The result of calculating the $\triangle E_{i} / E_{d i}$ of insensitive drug targets. Two combination (5-LOX, LTA4H and 5-LOX, 12-LOX) can satisfy $\triangle E_{i} / E_{d i} \leq 1$

by drugs in a combinatorial way to realize the transition from the disease to the normal state. The result is partially consistent with the biological findings because 5-LOX is a standard drug target among the key enzymes involved in the network responsible for generating inflammation mediators [17]. More importantly, some studies also support that the two combinations of 5-LOX, LTA4H and 5-LOX, 12-LOX are feasible drug target combinations in AA metabolic network [26, 27].

\section{Identifying the synergistic combinations of sensitive and insensitive drug targets}

We have determined that four among all eight drug targets are sensitive and the other four are insensitive. So, there are 16 pairs of combinations of sensitive and insensitive drug targets totally. For the sensitive drug targets, we set $\alpha=0.8$ and $\alpha$ is the constant in formula (3). In other words, the quantity of perturbation or drug to the sensitive target needed can be decreased $20 \%$ after the auxiliary perturbation to the insensitive target is performed. More exactly, synergistic drug target combination means $\triangle E_{i} / E_{d i} \leq 1$ for the insensitive drug target and $\left(\triangle E_{j}\right)^{1} /\left(\triangle E_{j}\right)^{2}=0.8$ for the sensitive drug target.

After calculating, there are 12 pairs of drug target combinations which can satisfy above conditions, as shown in Fig. 4. There are thee combinations have been proven to be viable by the corresponding biological experiments, including the combination of 15-LOX and PLA2 [28], combination of PLA2 and LTA4H [29], and combination of PLA2 and 5-LOX [30]. Especially, it has been found that pathway including PLA2 and LTA4H in
AA metabolic network is very important for traditional Chinese medicine anti-inflammatory herbal formulae and the treatment of cancers $[29,31,32]$.

\section{Discussion}

Due to the complexity nature of many diseases and the rising drug resistance, drug combination is becoming the standard treatment of many complex diseases. In this paper, we present a strategy to identify effective drug target combinations. Different from existing methods, the proposed strategy aims to identify effective drug target combinations from the perspective of network or systems biology. First, we select the enzymes as individual drug targets which can induce a transition from a disease state to a desired state when they are perturbed alone. Then, we divide them into sensitive and insensitive drug targets. Finally, we identify the synergistic and antagonistic combinations of all two types drug targets.

For the sensitive drug targets, we judge the synergistic and antagonistic of combination by the method of CI. For the combination of insensitive drug targets, two pairs of combination can restore the network to the normal state under our condition, and these combinations may be of great significance in biomedicine. For example, in the processing of disease treatment, when two drugs are used alone, they can not cure the disease, but when the two drugs are used simultaneously, the disease can be cured or the effect can be significantly improved. For the combination of sensitive and insensitive drug targets, it can reveal the intrinsic mechanism of the treatment of some diseases. For example, a single drug interventions for a 


\begin{tabular}{|c|c|c|c|c|c|c|c|c|}
\hline $\begin{array}{l}\text { Insensitive } \\
\text { (Enzyme 2) }\end{array}$ & \multicolumn{2}{|c|}{ 15-LOX } & \multicolumn{2}{|c|}{ 5-LOX } & \multicolumn{2}{|c|}{ LTA4H } & \multicolumn{2}{|c|}{ 12-LOX } \\
\hline $\begin{array}{l}\text { Sensitive } \\
\text { (Enzyme 1) }\end{array}$ & $\frac{\left(\frac{\Delta \mathrm{E}_{1}}{\mathrm{E}_{\mathrm{d} 1}}\right)^{1}}{\left(\frac{\Delta \mathrm{E}_{1}}{\mathrm{E}_{\mathrm{d} 1}}\right)^{2}}$ & $\frac{\Delta E_{2}}{E_{d 2}}$ & $\frac{\left(\frac{\Delta \mathrm{E}_{1}}{\mathrm{E}_{\mathrm{d} 1}}\right)^{1}}{\left(\frac{\Delta \mathrm{E}_{1}}{\mathrm{E}_{\mathrm{d} 1}}\right)^{2}}$ & $\frac{\Delta \mathrm{E}_{2}}{\mathrm{E}_{\mathrm{d} 2}}$ & $\frac{\left(\frac{\Delta \mathrm{E}_{1}}{\mathrm{E}_{\mathrm{d} 1}}\right)^{1}}{\left(\frac{\Delta \mathrm{E}_{1}}{\mathrm{E}_{\mathrm{d} 1}}\right)^{2}}$ & $\frac{\Delta \mathrm{E}_{2}}{\mathrm{E}_{\mathrm{d} 2}}$ & $\frac{\left(\frac{\Delta \mathrm{E}_{1}}{\mathrm{E}_{\mathrm{d} 1}}\right)^{1}}{\left(\frac{\Delta \mathrm{E}_{1}}{\mathrm{E}_{\mathrm{d} 1}}\right)^{2}}$ & $\frac{\Delta E_{2}}{E_{d 2}}$ \\
\hline CYP4F3 & 0.8 & 1.58 & 0.8 & 4.36 & 0.8 & 4.42 & 0.8 & 43.44 \\
\hline PLA2 & 0.8 & 0.995 & 0.8 & 1 & 0.8 & 1 & 0.8 & 1 \\
\hline PHGPX & 0.8 & 1.545 & 0.8 & 0.623 & 0.8 & 0.822 & 0.8 & 0.734 \\
\hline TXAS & 0.8 & 1 & 0.8 & 0.999 & 0.8 & 0.442 & 0.8 & 1 \\
\hline
\end{tabular}

Fig. 4 The combination of sensitive drug targets and insensitive drug targets. The red numbers represent the synergistic combinations

disease have reduced or no effect due to the rapid drug resistance response, however the joint intervention of two or more drugs show better efficacy and lower drug resistance. Similar situation can be often seen in the treatment of diseases. In many cases, although a single drug can cure a disease, the simultaneous intervention of multiple drugs not only can increase the efficacy but also can reduce the side effects of drugs.

In the part of identifying the synergistic and antagonistic combinations of the sensitive drug targets by computing $\mathrm{CI}$, for any one pair of combination, we mark one drug target with $\mathrm{A}$ and another with $\mathrm{B}$. In the process of calculating $\mathrm{CI}$, we first keep the concentration of A unchanged, and then adjust the concentration of $\mathrm{B}$ to restore the disease network to a normal state. Then we keep the concentration of $\mathrm{B}$ unchanged and adjust the concentration of A to restore the disease network to normal. We can get the same result in the two methods. We give one example of the combination of PLA2 and CYP4F3 in Fig. 5.

\section{Conclusion}

In this paper, we provide a new strategy to find the potential effective drug target combinations of all three types of drug target combinations involved in our paper. Among the combinations of drug targets we have screened, there are five combinations have been proven to be viable by the corresponding biological experiments, including the combination of 5-LOX and 12-LOX [26], combination of 5-LOX and LTA4H [27], combination of 15-LOX and PLA2 [28], combination of PLA2 and LTA4H [29, 31, 32] and combination of PLA2 and 5-LOX [30]. What's more, if we can combine the price and doses of drugs, we can have a better optimization. Although we mainly apply the proposed strategy to screen the effective combinations of two drug targets, the strategy can also be used to screen effective combinations of multiple drug targets in a straightforward manner.

\section{Methods}

\section{Construction of the AA metabolic network model in human} PMN

On the basis of the AA metabolic network, a set of ODEs were constructed to describe cell behavior of inflammation in human PMN(see details in Supplementary information). The ode $45 \mathrm{~s}$ routine of Matlab was used to integrate the ODEs. Michaelis Menten equations (Eq.3) were used to describe enzyme catalytic reactions in the network:

$$
\frac{d[S]}{d t}=\frac{K_{c a t}\left[E_{t}\right][S]}{K_{m}+[S]}
$$

where $[S]$ is the concentration of the substrate, $\left[E_{t}\right]$ is the total concentration of enzyme, $K_{c a t}$ is turnover number, and $K_{m}$ is the Michaelis-Menten constant.

If competitive reversible inhibitors are involved in the catalysis, the equation is:

$$
\frac{d[S]}{d t}=\frac{K_{c a t}\left[E_{t}\right][S]}{K_{m}\left(1+\frac{[I]}{K_{i}}\right)+[S]}
$$

where $[I]$ is the concentration of inhibitor and $K_{i}$ is the inhibition constant, which is defined as:

$$
K_{i}=\frac{[E][I]}{[E I]}
$$




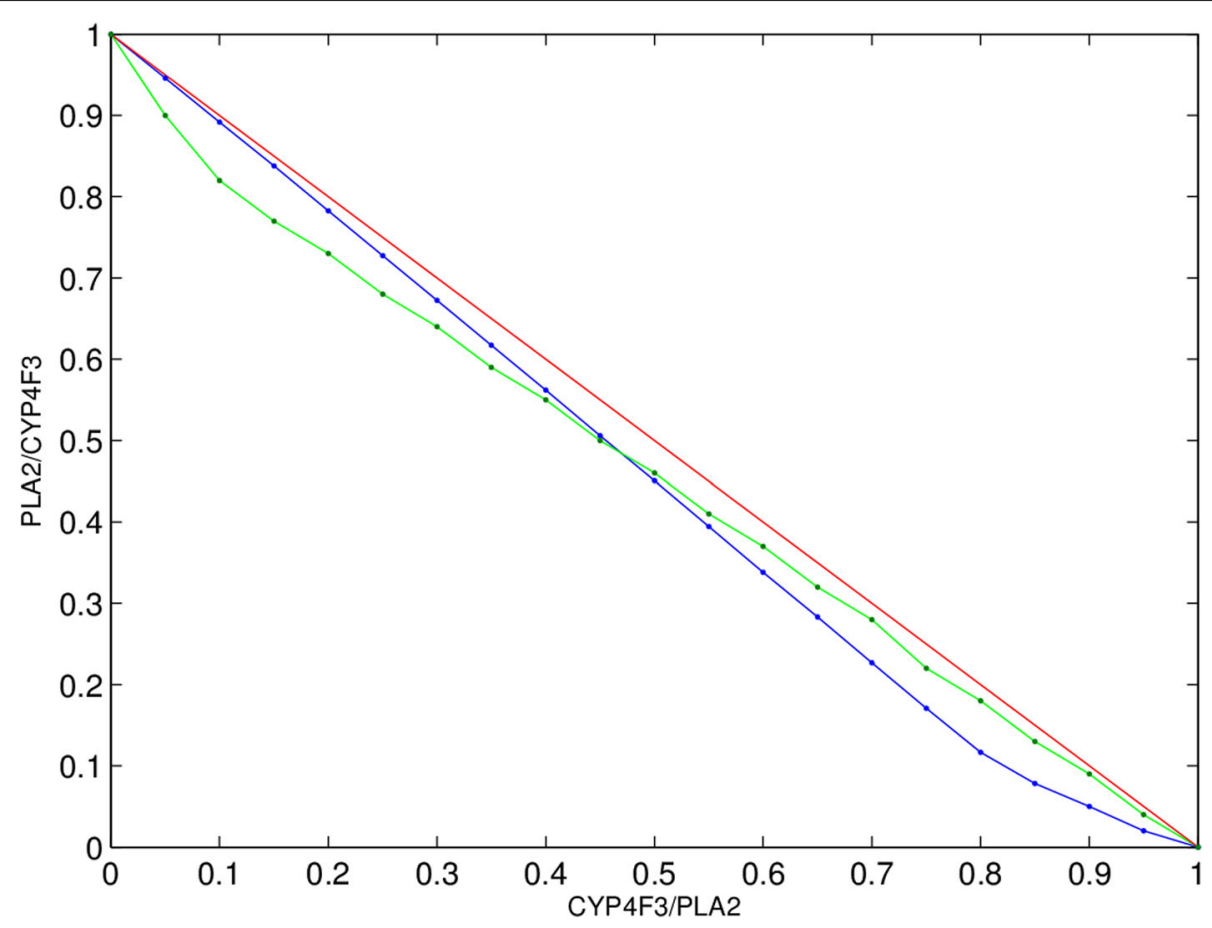

Fig. 5 The $\mathrm{Cl}$ isobolograms of PLA2 and CYP4F3

If the inhibitors are irreversible, we assume the enzymes would decay according to the following equation:

$$
\frac{d[E]}{d t}=-K[E][I]
$$

where $\mathrm{K}$ is a constant.

When activators are involved in the catalysis, we use the following equation:

$$
\frac{d[S]}{d t}=\frac{K_{c a t}(1+([A] / K I))\left[E_{t}\right][S]}{K_{m}+[S]}
$$

where $[A]$ is the concentration of activator and $K I$ is a constant.

When up regulation occurred though transcription, we described its effect with the following equation:

$$
\frac{d[E]}{d t}=\frac{k[g]^{2}}{[g]^{2}+[k]^{2}}
$$

where $[g]$ is the concentration of the metabolite up regulating the transcription of the enzyme, $K$ and $k$ are constants.

\section{Additional files}

Additional file 1: The ordinary differential equations of AA metabolic network was developed. A series of ODEs was established to simulate unicellular behavior, which included 24 initial concentrations and 45 reaction constants. (PDF $130 \mathrm{~kb}$ )

Additional file 2: A total of 23 reaction constants was taken from experimental values, while the others were obtained by fitting the calculated production of LTB4. (PDF $119 \mathrm{~kb}$ )

\section{Abbreviations}

AA: Arachidonic acid; Cl: Combination index; EC: Endothelial; LTs: Leukotrienes; ODEs: Ordinary differential equations; PGs: Prostaglandins; PLT: Platelet; PMNs: Human polymorphonuclear leukocytes; TCM: Traditional Chinese medicines

\section{Acknowledgments}

None.

\section{Funding}

This work was supported by the National Science Foundation of Shanghai (Grant No. 17ZR1410800). Publication costs are also funded by the National Science Foundation of Shanghai (Grant No. 17ZR1410800).

\section{Availability of data and materials}

The data and materials generated and/or analysed during the current study are available.

\section{About this supplement}

This article has been published as part of BMC Bioinformatics Volume 20 Supplement 7, 2019: Selected papers from the 12th International Conference on Computational Systems Biology (ISB 2018). The full contents of the supplement are available online at https://bmcbioinformatics.biomedcentral. com/articles/supplements/volume-20-supplement-7.

\section{Authors' contributions}

Min Luo, Jianfeng Jiao and Ruiqi Wang conceived the study. Min Luo, Jianfeng Jiao and Ruiqi Wang performed the numerical experiments and theoretical analysis. We confirm that all authors have approved the manuscript for submission.

Ethics approval and consent to participate

Not applicable.

\section{Consent for publication}

Not applicable.

\section{Competing interests}

The authors declare that they have no competing interests. 


\section{Publisher's Note}

Springer Nature remains neutral with regard to jurisdictional claims in published maps and institutional affiliations.

\section{Published: 1 May 2019}

\section{References}

1. Yoon BJ. Enhanced stochastic optimization algorithm for finding effective multi-target therapeutics. Bmc Bioinformatics. 2011;12(1):1-11.

2. Lindsay MA. Target discovery. Nat Rev Drug Discov. 2003;2(10):831-8.

3. Yang K, Bai HJ, Ouyang Q, Lai LH, Tang C. Finding multiple target optimal intervention in disease related molecular network. Mol Syst Biol. 2008;4:228.

4. Csermely P, Agoston V, Pongor S. The efficiency of multi-target drugs: the network approach might help drug design. TRENDS Phamacol Sci. 2005;26:178-82

5. Frantz S. Drug discovery: playing dirty. Nature. 2005;437:942-3.

6. Chou TC. Theoretical Basis, Experimental Design, and Computerized Simulation of Synergism and Antagonism in Drug Combination Studies. Pharmacol Rev. 2006;58(3):621-80.

7. Kitano H. A robustness-based approach to systems-oriented drug design. Nat Rrv Drug Discov. 2007:6:202-10.

8. Zhao XM, Iskar M, Zeller G, et al. Prediction of Drug Combinations by Integrating Molecular and Pharmacological Data. PLoS Comput Biol. 2011;7(12):e1002323.

9. Wang YY, Xu KJ, Song J, et al. Exploring drug combinations in genetic interaction network. BMC Bioinformatics. 2012;13(7 Supplement):0-0.

10. Zhang XD, Song J, Bork $P$, et al. The exploration of network motifs as potential drug targets from post-translational regulatory networks. Sci Rep-UK. 2016;6(1):20558.

11. Wu Z, Zhao XM, Chen L. A systems biology approach to identify effective cocktail drugs. Bmc Syst Biol. 2010;4 Suppl 2(S2):S7.

12. Gu Z, Steinmetz LM, Gu X, et al. Role of duplicate genes in genetic robustness against null mutations. Nature. 2003;421(6918):63-6.

13. Schoeberl B, Eichlerjonsson C, Gilles ED, et al. Computational modeling of the dynamics of the MAP kinase cascade activated by surface and internalized EGF receptors. Nat Biotechnol. 2002;20(4):370.

14. Rajasethupathy P, Vayttaden SJ, Bhalla US. Systems modeling: a pathway to drug discovery. Curr Opin Chem Biol. 2005;9(4):400-6.

15. Sung $M H$, Simon $R$. In silico simulation of inhibitor drug effects on nuclear factor-kappaB pathway dynamics. Mol Pharmacol. 2004;66(1):70-5.

16. Hwang WC, Zhang A, Ramanathan M. Identification of information flow-modulating drug targets: a novel bridging paradigm for drug discovery. Clin Pharmacol Ther. 2008;84(5):563-72.

17. Yang K, Ma W, Liang H, et al. Dynamic Simulations on the Arachidonic Acid Metabolic Network. Plos Comput Biol. 2007;3(3):e55.

18. Wu Z, Zhao XM, Chen L. A systems biology approach to identify effective cocktail drugs. Bmc Syst Biol. 2010;4 Suppl 2(S2):S7.

19. Huang L, Jiang Y, Chen Y. Predicting Drug Combination Index and Simulating the Network-Regulation Dynamics by Mathematical Modeling of Drug-Targeted EGFR-ERK Signaling Pathway. Sci Rep-UK. 2017;7:40752.

20. Liu Y, Li S, Liu Z, et al. Bifurcation-based approach reveals synergism and optimal combinatorial perturbation. J Biol Phys. 2016;42(3):1-16.

21. Chou TC. Theoretical Basis, Experimental Design, and Computerized Simulation of Synergism and Antagonism in Drug Combination Studies. Pharmacol Rev. 2006;58(3):621-81.

22. Fitzgerald JB, Schoeberl B, Nielsen UB. orger PK: Systems biology and combination therapy in the quest for clinical efficacy. Nat Chem Bioc. 2006;2(9):458-66.

23. Sullivan LC, Chavera TA, Gao X, et al. Regulation of delta opioid receptor-mediated signaling and antinociception in peripheral sensory neurons by arachidonic acid-dependent 12/15-lipoxygenase metabolites. 1. 2017;362:jpet.117.241604.

24. Simmons PM, Salmon JA, Moncada S. The release of leukotriene B 4, during experimental inflammation. Biochem Pharmacol. 1983;32(8): 1353-9.

25. Araujo RP, Liotta LA, Petricoin EF. Proteins, drug targets and the mechanisms they control: the simple truth about complex networks. Nat Rev Drug Discov. 2007;6(11):871-80.

26. Reddy KK, Rajan VKV, Gupta A, et al. Exploration of binding site pattern in arachidonic acid metabolizing enzymes, Cyclooxygenases and Lipoxygenases. Bmc Res Notes. 2015;8(1):152.
27. He C, Wu Y, Lai Y, et al. Dynamic eicosanoid responses upon different inhibitor and combination treatments on the arachidonic acid metabolic network. Mol Biosyst. 2012;8(5):1585-94.

28. Eleftheriadis N, Poelman H, Leus NGJ, et al. Design of a novel thiophene inhibitor of 15-lipoxygenase-1 with both anti-inflammatory and neuroprotective properties. Eur J Med Chem. 2016;122:786-801.

29. Wei D, Jiang $X$, Zhou $L$, et al. Discovery of Multitarget Inhibitors by Combining Molecular Docking with Common Pharmacophore Matching. J Med Chem. 2008;51(24):7882-8.

30. Gu S, Yin N, Pei J, et al. Understanding Traditional Chinese Medicine Anti-inflammatory Herbal Formulae by Simulating Their Regulatory Functions on the Human Arachidonic Acid Metabolic Network. Mol Biosyst. 2013;9(7):1931-8.

31. Gu Y, Zhang X, Chen YK, et al. Discover potential inhibitors of 5-LOX and LTA4H from Rhei Radix et Rhizoma, Notopterygii Rhizoma et Radix and Genitana Macrophyllae Radix based on molecular simulation methods. China J Chin Materia Medica. 2017;42(23):4494-502.

32. Sun Z, Sood S, Li N, et al. Involvement of the 5-lipoxygenase/leukotriene A4 hydrolase pathway in 7,12-dimethylbenz[a]anthracene (DMBA)induced oral carcinogenesis in hamster cheek pouch, and inhibition of carcinogenesis by its inhibitors. Carcinogenesis. 2006;27(9):1902-8.

\section{Ready to submit your research? Choose BMC and benefit from}

- fast, convenient online submission

- thorough peer review by experienced researchers in your field

- rapid publication on acceptance

- support for research data, including large and complex data types

- gold Open Access which fosters wider collaboration and increased citations

- maximum visibility for your research: over $100 \mathrm{M}$ website views per year

At $\mathrm{BMC}$, research is always in progress.

Learn more biomedcentral.com/submissions 